\title{
Signatures of multiple episodes of AGN activity in the core of Abell 1795 $\star$
}

\author{
G. Kokotanekov ${ }^{1}$, M. W. Wise ${ }^{2,1}$, M. de Vries ${ }^{1}$, and H. T. Intema ${ }^{3}$ \\ 1 Anton Pannekoek Institute for Astronomy, University of Amsterdam, Postbus 94249, 1090 GE Amsterdam, The Netherlands \\ e-mail: g.d.kokotanekov@uva.nl \\ 2 Netherlands Institute for Radio Astronomy (ASTRON), Postbus 2, 7990 AA Dwingeloo, The Netherlands \\ e-mail: wise@astron.nl \\ ${ }^{3}$ Leiden Observatory, Leiden University, Niels Bohrweg 2, 2333 CA Leide, The Netherlands
}

Received 12 April 2018 / Accepted 2 July 2018

\begin{abstract}
In this paper we analyze AGN activity signatures in the rich nearby galaxy cluster Abell 1795 aiming to confirm and characterize the long-term feedback history in the system. We combine radio observations at 610 and $235 \mathrm{MHz}$ from the Giant Metrewave Radio Telescope (GMRT) with $3.4 \mathrm{Msec} \mathrm{X}$-ray data from the Chandra Observatory. Extracting radial temperature profiles, as well as $\mathrm{X}$-ray and radio surface brightness profiles in three directions showing major morphological disturbances, we highlight the signatures of activity in the system. For the first time we observe radio emission corresponding to the NW X-ray depression, which provides evidence in favor of the classification of the depression as a cavity. We identify two other X-ray cavities situated NW and SW of the AGN. While the central radio emission corresponding to the inner cavities shows a flatter spectral index, the radio extensions associated with the farthest X-ray cavities consist of aged plasma. All observed signatures both in radio and X-ray are consistent with several consecutive episodes of AGN activity, which gave rise to the observed morphology NW and SW from the core. In the southern region, we confirm the cooling wake hypothesis for the origin of the long tail. The deep X-ray data also allows us to distinguish significant distortions in the tail's inner parts, which we attribute to the activity of the AGN.
\end{abstract}

Key words. galaxies: clusters: general - galaxies: clusters: individual: Abell 1795 - galaxies: clusters: intracluster medium radio continuum: general - X-rays: galaxies: clusters - methods: observational

\section{Introduction}

Central active galactic nuclei (AGN) play a crucial role in the evolution of galaxy cluster cores providing energy that regulates both the growth of the black hole and the formation of stars in the surrounding galaxy (Silk \& Rees 1998; Gebhardt et al. 2000; Ferrarese \& Merritt 2000). In the classical model, symmetric cavities observed in X-rays are blown into the intracluster medium (ICM) on either side of the brightest cluster galaxy (BCG) by the expanding radio lobes generated by the central AGN. For many systems, these X-ray cavities are observed to be filled with radio emitting plasma (Fabian et al. 2000; McNamara et al. 2000), further supporting this model. Deeper X-ray observations and new radio data, however, show that establishing this simple correspondence over longer feedback timescales can be more difficult.

Deep X-ray observations of the cores of nearby relaxed galaxy clusters have revealed a wide range of irregular surface brightness features not easily identified as simple, spherical cavities (e.g., Perseus: Boehringer et al. 1993; Fabian et al. 2006, 2011; Zhuravleva et al. 2015 and Hydra A: McNamara et al. 2000; Nulsen et al. 2002; Wise et al. 2007). In a number of these systems, low-frequency radio observations have shown diffuse, extended steep-spectrum emission presumably representing the

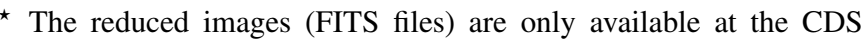
via anonymous ftp to cdsarc.u-strasbg.fr (130.79.128.5) or via http://cdsarc.u-strasbg.fr/viz-bin/qcat?J/A+A/618/ A152
}

signatures from older AGN outbursts (M 87: de Gasperin et al. 2012, Perseus: Burns et al. 1992; Sijbring 1993, Hydra A: Wise et al. 2007, 2A 0335+096: Kokotanekov et al. 2017). At radio frequencies below $1 \mathrm{GHz}$, however, the close morphological correspondence between the observed X-ray structures and radio emission seen at higher frequencies is not always apparent. To understand the impact of feedback over the lifetime of the cluster, it is important to determine how these complicated morphologies map to the long-term AGN activity in the core.

Abell 1795 (hereafter A1795) is an excellent example of a source exhibiting such complicated structures. It is a rich, nearby cluster of galaxies $(z=0.063$, with an angular scale of $1.22 \mathrm{kpc}$ per arcsec) that hosts the powerful Fanaroff-Riley type I (FR I) radio source 4C 26.42. While classified as a relaxed, cool-core cluster because of its regular morphology on larger scales (Buote \& Tsai 1996), A1795 shows evidence of a variety of activity throughout the cluster core. Deep Chandra data have revealed a myriad of structures (Markevitch et al. 2001; Ettori et al. 2002; Walker et al. 2014, 2017; Crawford et al. 2005; Ehlert et al. 2015) not easily described by a simple feedback scenario involving only a single outburst.

One such structure, a large $r \sim 34 \mathrm{kpc}$ depression to the northwest of the central AGN, was previously identified in Chandra data (Crawford et al. 2005) and classified as a cavity by Walker et al. (2014) based on its morphology. Despite its suggestive X-ray properties, however, previous studies at higher frequencies have failed to detect radio emission associated with the cavity. The cavity also lacks a counterpart on the opposite side of the 
cluster as well as other typical observational signatures normally associated with X-ray cavities, such as evidence of abundance gradients or optical filaments created as the cavity rises in the cluster atmosphere. Taken together, this lack of additional evidence has made it difficult to definitively classify the NW structure as a traditional cavity associated with an outburst.

The core of A1795 also contains a dramatic filament of cool gas extending over $\sim 50 \mathrm{kpc}$ to the south of the central $\mathrm{cD}$ galaxy (Fabian et al. 2001) and coincident with an optical $\mathrm{H} \alpha$ filament (Cowie et al. 1983). Several theories for the origin of this filament have been proposed, but Fabian et al. (2001) have argued that the most likely origin is that the feature represents a "cooling wake" produced as the ICM cools behind the central galaxy as it moves relative to the surrounding gas. Alternatively, this filament may consist of cold gas from the central galaxy which has been stripped out by dynamical friction due to the motion of the cD galaxy. Regardless of the exact formation mechanism, the presence of such a dramatic feature and its implications for the relative motions of the gas and central galaxy clearly point to a more complicated evolution of the core region in A1795 than can be explained by a simple AGN outburst model.

Finally, Markevitch et al. (2001) have shown that the inner $60 \mathrm{kpc}$ core of A1795 exhibits a surface brightness edge, which they interpret as a "cold front" or contact discontinuity caused by cool gas in the core sloshing in the central potential well of the cluster. In this scenario, the cool gas interior to the surface brightness edge is believed to be sloshing roughly north to south and is observed at or near the point of maximum displacement. The observed filament of cool material is then a cooling wake produced, not by motions of the $\mathrm{cD}$ galaxy in the potential well itself, but rather by the movement of the sloshing gas. The cumulative impact of the larger scale sloshing motion of the cool core and AGN activity (Ehlert et al. 2011; Blanton et al. 2011 ) is evoked to explain the profoundly disturbed morphology observed in the cool core of A1795.

Altogether these features imply a dynamic environment in the core of A1795 over the last several hundred Myr, and one that does not easily conform to the simple picture of an AGN-driven outburst. In this paper, we present a joint analysis for the core of A1795 combining a deep, 3.4 Msec archival X-ray dataset from Chandra and new low-frequency GMRT observations at 235 and $610 \mathrm{MHz}$. We describe the X-ray and radio data along with the data reduction process in Sect. 2. Sections 3 and 4 compare the morphologies of the different features revealed in the deep Chandra X-ray maps with the low-frequency radio structures observed in the core of A1795. We perform a multiwavelength sector analysis in Sect. 5 to isolate various features of interest in the core and examine the underlying thermodynamic properties of the gas in an effort to constrain the integrated feedback history over the last few hundred Myr. The properties of the cold filament are presented in Sect. 6 in the context of current theories for its origin. The paper concludes in Sect. 7 with a summary of our analysis and a discussion of the implications of our results.

\section{Data reduction}

\subsection{X-ray data}

We have extracted and reprocessed existing archival Chandra data for A1795, which has been used extensively as a Chandra calibration and reference target since launch and, consequently, has one of the deepest accumulated exposures of any cluster target. In this work, we analyze over $3.4 \mathrm{Msec}$ of Chandra data, consisting of 148 individual ObsIDs taken between December
1999 and November 2016. Most of these data were observed as part of the Chandra calibration program and include 33 and 115 pointings taken with the ACIS-S and ACIS-I detectors, respectively. In order to assess the calibration properties of individual ACIS CCDs, the aimpoints for many of these observations also vary. When combined, these variations result in a somewhat broader point spread function (PSF) across the core of A1795. Our features of interest are significantly larger than the Chandra PSF, so this broadening should have no appreciable effect on the analysis presented here.

Each ObsID was reprocessed using CIAO 4.8 (Fruscione et al. 2006) and CALDB 4.7.2 to apply the latest gain and other calibration corrections. Standard filtering was applied to the event files to remove bad grades and pixels. Additionally, all ObsIDs were examined for contamination due to strong background flares and filtered to remove the effected time intervals using the CIAO tool deflare. These filtered event files were also corrected for the presence of "out-of-time" events detected during the frame readout process using the readout_bkg routine. The final combined exposure time after all corrections was $3.45 \mathrm{Msec}$.

ACIS blank-sky event files from the CALDB were used to construct background event files for each ObsID. The blank-sky event files were reprocessed in a manner similar to the observation data to apply the latest calibrations. The CALDB ACIS blank-sky event files have already been screened for background flares so no additional filtering for flares was applied. Finally, the exposure times for each blank-sky event file were scaled to match the observed background rate in the $10-12 \mathrm{keV}$ band for each ObsID. These scaled background event files were used in all subsequent spectral analyses discussed below.

\subsubsection{Surface brightness maps}

An X-ray mosaic of the surface brightness in A1795 was constructed by reprojecting all event files to a common tangent point on the sky before combining them. The event file for each ObsID was reprojected to match the coordinate frame of the reference ObsID 493. To correct for variations in the exposure and other instrumental artifacts across the field, instrument and exposure maps were constructed for each ObsID in the $0.5-7.0 \mathrm{keV}$ energy range. We have accounted for the energy dependence of the instrument response by utilizing a spectral weighting determined by fitting the integrated spectrum for the central $2^{\prime}$ region of A1795. Bright point sources were excised from the region and the resulting total spectrum was fit with a single temperature, APEC (Smith et al. 2001) thermal model multiplied by foreground Galactic absorption. The Galactic absorption was fixed to a value of $N_{\mathrm{H}}=1.2 \times 10^{20}$ as determined by the LAB Survey of Galactic HI (Kalberla et al. 2005). This model gives a good fit to the data with a reduced $\chi^{2}=1.1$ and best-fit temperature and metallicity of $k T=5.7 \mathrm{keV}$ and $Z=0.41$, respectively.

The resulting spectrally weighted exposure maps were combined into a single mosaic after reprojection to the common tangent point. Similarly, a map of the background surface brightness was constructed by reprojecting the scaled, blank-sky event files for each ObsID and combining them. To obtain the final, flat-fielded, surface brightness map, we subtracted the total background map from the source counts mosaic and divided by the exposure map for the field. The resulting background-subtracted, exposure-corrected surface brightness mosaic for the core of A1795 in the energy range $0.5-7.0 \mathrm{keV}$ is shown in Fig. 1. Various features of interest are described in Sect. 3.

Although several morphological features like the cool tail of gas to the south are visible in Fig. 1, the overall surface brightness 


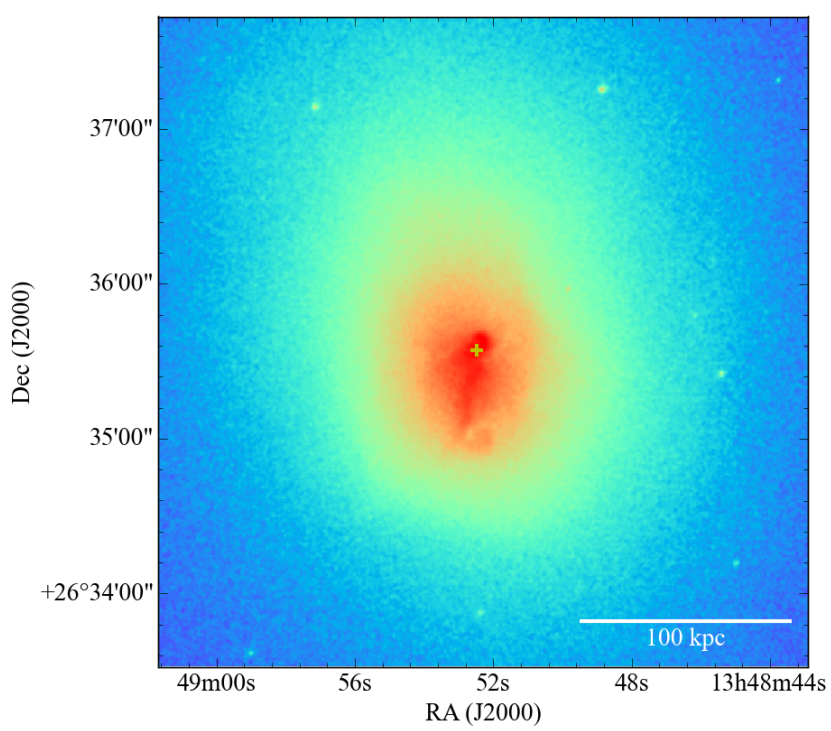

Fig. 1. Exposure-corrected, background-subtracted $0.5-7.0 \mathrm{keV}$ surface brightness mosaic for the core of A1795 constructed from the existing 3.4 Msec Chandra data. The field measures $4 \times 4 \operatorname{arcmin}^{2}$ and has been smoothed with a $\sigma=3^{\prime \prime}$ Gaussian. The cross indicates the position of the center of the radio source.

distribution in the core of A1795 is quite smooth and other features are less apparent. To enhance the visibility of these other features, we constructed an X-ray residual map using a radial unsharp masking (RUM) technique. This technique is described in Wise et al. (2018) and is similar to traditional unsharp masking, except rather than computing the difference between the original image when convolved with two different 2D smoothing kernels, the RUM algorithm applies a single 1D smoothing kernel radially from the image center. A Radon transform (Radon 1986) was applied to the original surface brightness map to convert from $(x, y)$ image coordinates to $(r, \Theta)$ coordinates, where $r=0$ is taken to be the center of the radio source. The resulting transformed image was then smoothed with a 1D Gaussian kernel with $\sigma=8$ arcsec along the radial direction. Finally, the smoothed image in $(r, \Theta)$ coordinates is transformed back to the $(x, y)$ image plane and subtracted from the original surface brightness map. This technique has the advantage of being less prone to creating circularly symmetric bowl features due to the mismatch between the two 2D smoothing kernels used in traditional unsharp masking techniques (Wise et al. 2018). The resulting residual image is shown in Fig. 2.

\subsubsection{Spectral fitting}

In the subsequent analysis, we perform spatially resolved spectral fitting to determine the underlying thermodynamic properties of the gas in the core of A1795. We consider both 2D spectral mapping and radial profiles extracted in different sectors of interest as determined by the morphological features seen in the surface brightness maps. For the 2D spectral maps, we used the contbin algorithm developed by Sanders (2006) to define a set of extraction regions covering a $10 \times 10 \mathrm{arcmin}^{2}$ region centered position of the central AGN in A1795. A position for the AGN of (RA 13:48:52.473, Dec +26:35:34.385) was used based on the $1.4 \mathrm{GHz}$ VLA map of Bîrzan et al. (2008). The regions were defined to have a constant signal-to-noise ratio (S/N) of 100 after background subtraction, and therefore roughly the same errors on the derived fit parameters.

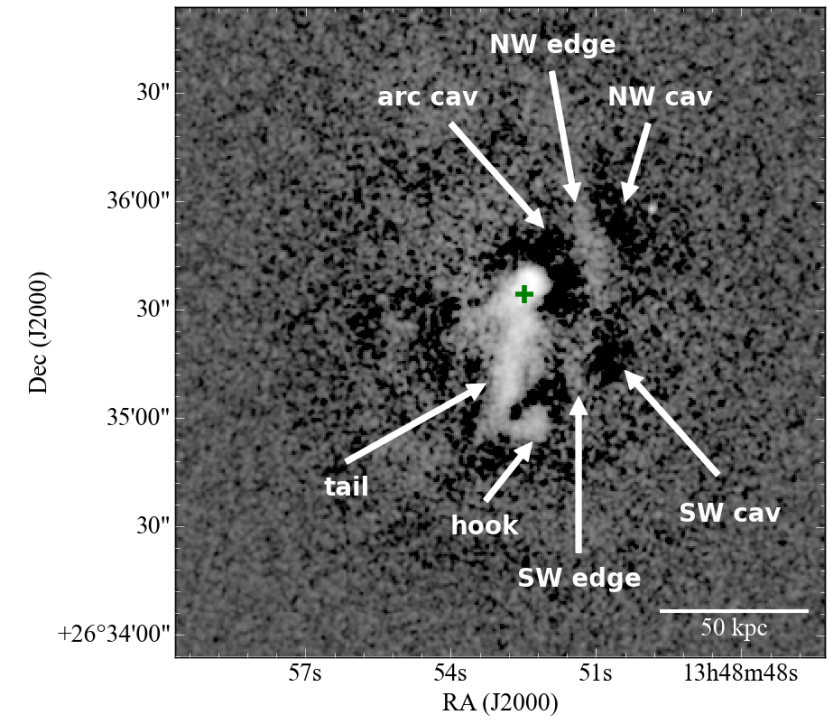

Fig. 2. Chandra X-ray surface brightness residual map of A1795 in the $0.5-7 \mathrm{keV}$ band. The image was produced by applying a radial unsharp masking (RUM) algorithm to the image in Fig. 1 as described in the text. The cross indicates the position of the core of the radio source. Various features of interest in the core of A1795 are labeled.

Radial profiles were constructed by defining a set of annular extraction regions centered on the position of the AGN and adaptively adjusted in width so as to contain a given $\mathrm{S} / \mathrm{N}$ after background subtraction. Unless noted otherwise, a minimum $\mathrm{S} / \mathrm{N}$ of 100 was used and annuli out to a radius of 5 arcmin were defined. We defined annular extraction regions encompassing the entire cluster profile and a set of sector profiles limited to a given angular range and designed to isolate features of interest along a given radial direction through the core of A1795. These radial sectors and their correspondence to specific surface brightness features are discussed in Sect. 5.

The spectra in a given region were extracted using the CIA0 4.8 tool specextract. This tool produces source and background spectra, an instrument response (RMF) file, and an effective area (ARF) file, all of which are used in the spectral fitting analysis. This set of spectral files is produced for every extraction region for each CCD from each ObsID. The resulting set of spectral files from all ObsIDs contributing to a given extraction region are then combined into a single composite set using the combine_spectra tool in the default way. After combination, we adjust the total exposure time of each extraction region to correct for the fact that regions that fall on different chips within the same ObsID are counted twice when combine_spectra adds them, which incorrectly increases the total exposure time of the combined spectrum. The final set of merged spectral files for all extraction regions are then used as input to the spectral fitting analysis.

The ObsIDs for A1795 used in our analysis span a wide range in time in which the effective area of ACIS has significantly changed, and also include observations taken with both the ACIS-S and the ACIS-I detectors. The time changes and the intrinsic differences of the two types of CCDs predominatly effect the spectral response below $2.0 \mathrm{keV}$. The exposure-weighted averaging scheme employed by the CIAO combine_spectra tool effectively averages over these variations to produce a single composite ARF and RMF for a given region. When performing spectral fits with these composite ARFs and RMFs, there is the risk of deriving incorrect or biased results if the variations from the average are too large. We note, how- 

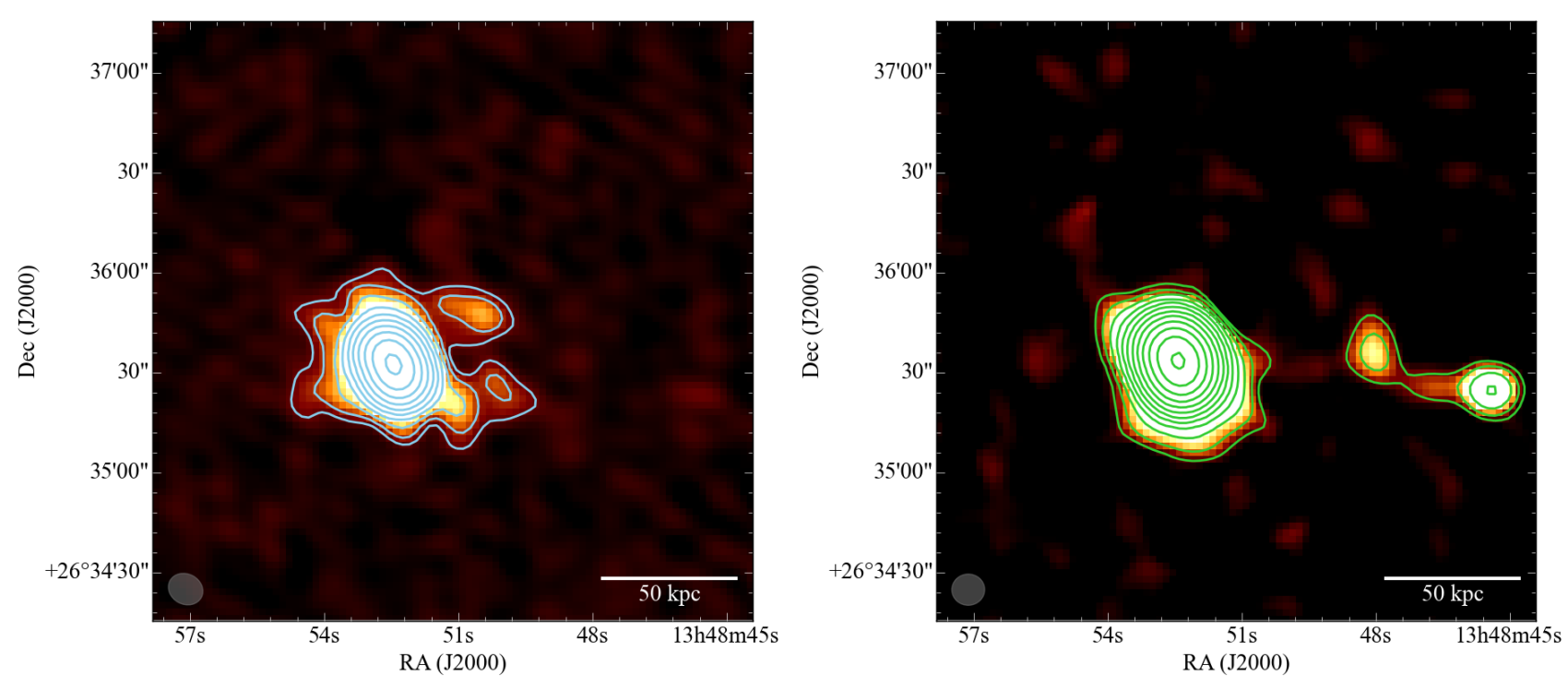

Fig. 3. GMRT maps of A1795. Left: radio map at $235 \mathrm{MHz}$ with resolution of $10.7 \times 9.1^{\prime \prime}$ and rms noise level of $0.8 \mathrm{mJy}^{\mathrm{m}}$ beam ${ }^{-1}$. Contours are drawn at $5 \sigma \times[1,2,4,8,16,32,64,128,256,512]$. Right: radio map at $610 \mathrm{MHz}$ with resolution of $9.9 \times 9.4^{\prime \prime}$ and noise level of $0.18 \mathrm{mJy}^{\mathrm{beam}}{ }^{-1}$. Contours are drawn at $3 \sigma \times[1,2,4,8,16,32,64,128,256,512,1024,2048]$.

ever, that for the range of hotter gas temperatures $(3-6 \mathrm{keV})$ in the core of A1795 these variations in the soft response of the telescope are expected to be less significant.

To check whether the composite ARFs and RMFs give results that are accurate enough, we separated the datasets into subsets of ObsIDs observed on ACIS-S and ACIS-I, respectively. These subsets were then each processed separately to produce summed spectra and composite ARFs and RMFs for ACIS-S and ACIS-I. The spectral analysis in the different sectors was then redone using a joint fit to the composite ACIS-S and ACISI spectra in each region. The resulting temperature profiles and other spectral fit parameters all agree with the original results based on the composite spectral files from all ObsIDs to within less than $5 \%$. Given that the typical measurement error on the derived spectral parameters is typically $5-10 \%$, we conclude that the composite spectral files are sufficient to derive the underlying spectral properties of the ICM in A1795.

All spectral analyses presented were performed using Sherpa in CIA0 4.8 over the $0.5-7.0 \mathrm{keV}$ energy range. Except where noted otherwise, all data were grouped to contain a minimum of 25 counts per bin and fit with a PHABS*APEC spectral model using the default $\chi^{2}$ fit statistic.

\subsection{Radio data}

We processed archival radio observations from the Giant Metrewave Radio Telescope (GMRT; Swarup 1991) on A1795 using the fully automated SPAM pipeline (Intema 2014), which incorporates correction for direction-dependent effects (Intema et al. 2009). The observations under project code 20_016 consist of simultaneous dual-frequency observations at 235 and $610 \mathrm{MHz}$, using bandwidths of 16.7 and $33.3 \mathrm{MHz}$, respectively. The total on-source time was $273 \mathrm{~min}$ (or $4.55 \mathrm{~h}$ ). Flux and bandpass calibrations were derived from 3C468.1 and 3C286, respectively, using flux models based on the work by Scaife \& Heald (2012). Phase calibrations were not derived from the secondary calibrators, but instead from (self-)calibration against the TGSS (Intema et al. 2017) in the case of $235 \mathrm{MHz}$, and against the $235 \mathrm{MHz}$ image in the case of the $610 \mathrm{MHz}$. During the pipeline processing about $50 \%$ of the data was removed because of non- functional antennas, radio-frequency interference (RFI), and bad ionospheric conditions. The output images and visibility data were post-processed to allow final imaging in CASA. For this, all sources outside a radius of $2^{\prime}$ from A1795 were subtracted from the visibility data, effectively removing all direction-dependent effects.

To image the GMRT data we used the interactive cleaning of CASA setting Briggs weighting scheme with a robust parameter of -0.2 for both frequencies. We imposed a Gaussian taper with outer $u v$-cut of $10^{\prime \prime}$ at $610 \mathrm{MHz}$ in order to highlight the large-scale structures and achieve comparable resolution with the $235 \mathrm{MHz}$ image. The end resolution of the $610 \mathrm{MHz}$ image is $9.9 \times 9.4^{\prime \prime}$ at an rms noise level of $0.18 \mathrm{mJybeam}^{-1}$. The $235 \mathrm{MHz}$ image has a resolution of $10.7 \times 9.1^{\prime \prime}$ and a noise level of $0.8 \mathrm{mJy}^{\text {beam }}{ }^{-1}$. The GMRT images are presented in Fig. 3.

We complement our study with published VLA maps at $1.4 \mathrm{GHz}$ in $A$-configuration (Bîrzan et al. 2008) and $C$ configuration (Giacintucci et al. 2014). The $A$-configuration image has an rms noise level of $70 \mu \mathrm{Jy}_{\text {beam }^{-1}}$. It is based on an observation with exposure of $174 \mathrm{~min}$ and a bandwidth of $50 \mathrm{MHz}$. The size of the FWHM of the synthesized beam is $1.2 \times 1.1^{\prime \prime}$. The resolution of the $C$-configuration map is $19 \times 16^{\prime \prime}$. The corresponding radio data was integrated for $130 \mathrm{~min}$ at a bandwidth of $6 \mathrm{MHz}$. The resulting noise level is $150 \mu \mathrm{Jy} \mathrm{beam}^{-1}$.

\section{X-ray morphology}

In this section we discuss the observed X-ray morphology in the core of A1795. We identify the most pronounced features of interest, which we study in detail in the subsequent sections.

\subsection{Large-scale structure}

A1795 has been extensively observed at X-ray wavelengths in the core and on larger scales. It has been shown that the structure of the core of A1795 exhibits a very smooth and circularly symmetric surface brightness distribution at a radius beyond $\sim 80 \mathrm{kpc}$ from the AGN (e.g., Ettori et al. 2002; Ehlert et al. 2015). In the surface brightness map presented in Fig. 1, we focus on the 
central region of A1795 within a radius of $150 \mathrm{kpc}$ centered on the central AGN. Inside a radius of $70-80 \mathrm{kpc}$, the structure of the ICM appears heavily disturbed. The cool tail is quite evident; it extends due south away from the core and appears to end with a hook-like structure that bends toward the west. We are also able to identify the inner rim of the large depression NW from the core studied by Walker et al. (2014).

These features and others are even more prominent in the $\mathrm{X}$-ray residual map presented in Fig. 2. In the NW direction we see a number of features including the large outer depression. Its inner rim is a distinct edge of bright emission situated $28 \mathrm{kpc} \mathrm{NW}$ from the core. The most pronounced region of the depression is situated at a distance of $\sim 40 \mathrm{kpc}$ NW from the center and appears elongated in the tangential direction. The NW depression has been classified as a cavity and studied in detail by Walker et al. (2014), who define its size based on the curvature of the concave NW edge. They measure a distance from the central galaxy to the center of the outer NW cavity of $54 \mathrm{kpc}$ and derive an age of $t_{\mathrm{s}}=41 \mathrm{Myr}$ based on the sound speed. Closer to the AGN we observe another depression surrounding the active core to the N, NW, and $\mathrm{W}$. This depression appears severely distorted and is located $\sim 15 \mathrm{kpc}$ from the center, being nested between the centrally peaked core of the X-ray surface brightness and the NW edge.

We also identify similar features, both an edge and outer Xray depression, to the $\mathrm{SW}$. The $\mathrm{SW}$ edge peaks $25 \mathrm{kpc}$ from the BCG. Thus the SW edge appears to be situated slightly closer to the core than the NW edge. The depression is situated outside of the SW edge, at a distance $33 \mathrm{kpc}$ from the AGN. These are new structures that have not been studied previously and, as we show in the following section, they are not artifacts from the construction of the residual map. We discuss these features in light of a possible feedback origin in Sect. 5.

The X-ray residual map clearly shows the cold tail to the south and confirms the finding from previous studies (e.g., Ehlert et al. 2015) that the cold tail is very confined. It stretches as far as $47 \mathrm{kpc}$ from the core, ending with a hook extending to the west. The new Chandra data, however, allow us to examine the substructure of the tail in greater detail than before. Our $\mathrm{X}$-ray residual map reveals small-scale inhomogeneities in its structures and lets us identify kinks in its direction of propagation. The properties of the cool tail are discussed in more detail in Sect. 6.

Crawford et al. (2005) identified a small-scale hole in the Xray emission locked between the hook and the end of the tail, centered at RA 13:48:52.7, Dec +26:35:01.8. Later this depression was analyzed by Walker et al. (2014) in an attempt to identify its origin. Our residual map reveals a continuation of this depression to the north, which is enclosed between the hook, the tail, and the SW edge. We defer the detailed analysis of the resulting irregularly shaped depression to future studies.

Although the eastern half of the X-ray map seems less rich in structure than the western side, the emission in this region is far from uniform. Our map reveals several ripples and irregularly shaped depressions in the region $\mathrm{E}$ and SE from the center. However, the observed features appear severely distorted and are not as easy to classify. Since these structures are not as distinct and we do not have enough evidence to identify their nature, we leave their analysis for future work.

\subsection{Temperature and pressure}

If the morphological features identified in the residual map are related to AGN activity, then the thermodynamic properties of the gas in the core should show evidence of the energy deposited into the ICM by the activity. To look for this evidence, we constructed temperature and pressure maps for the ICM in the center of A1795. As discussed in Sect. 2.1.2, we defined an adaptively sized grid of $2 \mathrm{D}$ extraction regions over the central $10 \times 10 \operatorname{arcmin}^{2}$ of A1795 and extracted summed spectra for all ObsIDs contributing to a given region. These spectra were then fit with a PHABS*APEC model to determine the projected gas temperature as a function of position in the core. The Galactic absorption was held fixed to a value of $N_{\mathrm{H}}=1.2 \times 10^{20}$ and the abundance was a free parameter in the model. The normalization of the fitted spectrum can be used to derive the particle number density, $n$, and when combined with the temperature map to construct a projected map of the gas pressure in the core. The projected temperature and pressure maps are presented in Fig. 4.

The temperature map shows the NW X-ray depression as a region of enhanced temperature compared to the ambient. The most pronounced temperature difference between the depression and the surrounding medium appears at the position of the NW edge. The dense NW edge clearly separates the $\sim 6 \mathrm{keV}$ gas within the cavity from the $\sim 5 \mathrm{keV}$ gas between the edge and the active core. The abrupt NW temperature jump has been analyzed by several authors (e.g., Markevitch et al. 2001; Ehlert et al. 2015; Walker et al. 2014). It has been shown that it is not consistent with a shock moving out since in this case the gas should be hotter behind the compressed region. The observed edge is more consistent with a cold front (Markevitch et al. 2001), due to its reversed temperature structure.

The overall large-scale temperature structure is consistent with previous publications. We see the cold front situated $\sim 80 \mathrm{kpc}$ south of the center reported by Markevitch et al. (2001). The cold tail is very pronounced in the temperature map appearing as a strip of material much colder than its surroundings.

The pressure morphology in the central $\sim 20 \mathrm{kpc}$ from the AGN is radially symmetric, and in this region we measure systematically higher values than at the central AGN. Outside this radius the pressure structure appears irregularly distributed. The map reveals large-scale extension of high pressure relative to the ambient stretching SE and NW. This symmetry in the NW-SE direction might be evidence of an older symmetric outflow due to the traditionally expected bipolar AGN ejection.

\section{Radio morphology}

In this section we discuss the structure in the core of A1795 observed at different radio frequencies. We describe the morphological features revealed by our new GMRT images at 235 and $610 \mathrm{MHz}$. We also include two previously published VLA observations at $1.4 \mathrm{GHz}$. The high-resolution map of Bîrzan et al. (2008) shows the inner radio lobes, while the lower-resolution image of Giacintucci et al. (2014) reveals the large-scale structure of the source. We discuss the radio lobes in the context of the pressure structure of the core, while the morphology observed in the map of Giacintucci et al. (2014) is compared with the structures observed with GMRT.

\subsection{VLA 1.4 GHz A-configuration}

The inner radio lobes of the central AGN have been studied using VLA observations at $8.2 \mathrm{GHz}$ and $1.4 \mathrm{GHz}$ (e.g., Ge \& Owen 1993; Bîrzan et al. 2008). In Fig. 5 we present the radio contours of the $A$-configuration $1.4 \mathrm{GHz}$ image from Bîrzan et al. (2008) overplotted on an enlarged section of our temperature map showing the active core and the cool tail. The observed 

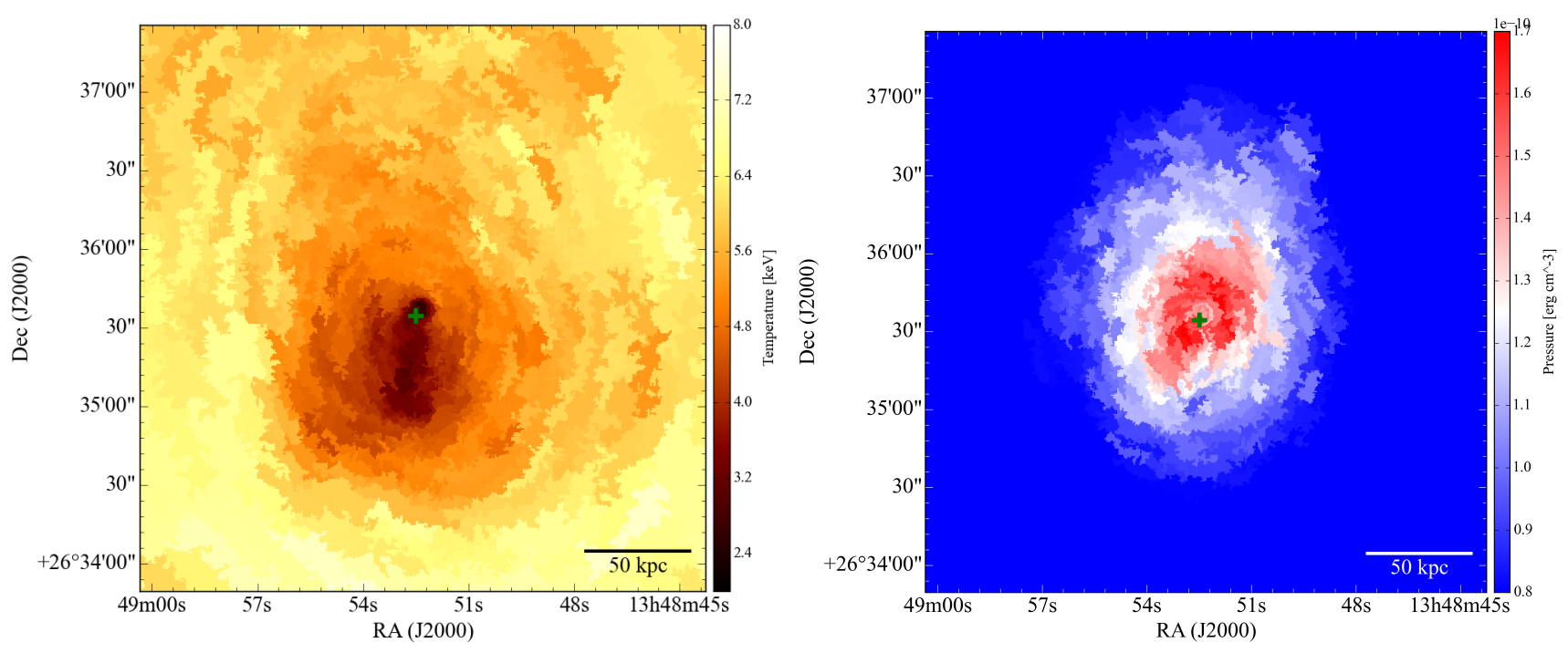

Fig. 4. Temperature (left) and pressure (right) maps of the core of A1795 within a radius of $130 \mathrm{kpc}$ from the AGN. Both maps are derived from Chandra imaging X-ray spectroscopy using regions with a $\mathrm{S} / \mathrm{N}$ of 100, as described in Sect. 2.1. The cross indicates the center of the radio source.

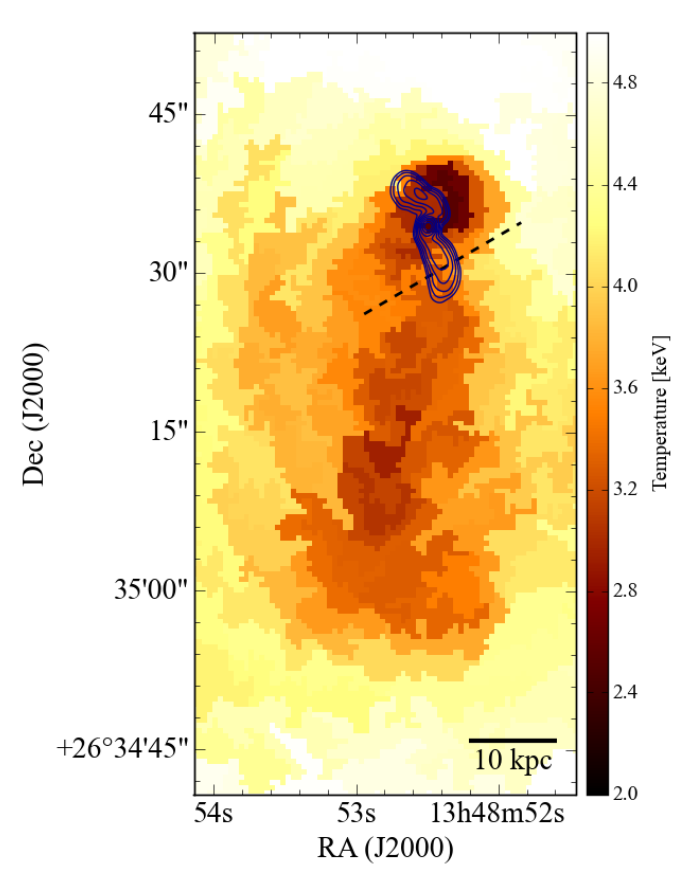

Fig. 5. Temperature map of the cold tail with radio contours at $1.4 \mathrm{GHz}$ from the VLA in A-configuration from Bîrzan et al. (2008). Contours are drawn at $10 \mathrm{mJy}_{\text {beam }}^{-1} \times[1,1.4,2,2.8,4,5.7,8,11]$. The black dashed line indicates the division between the inner and outer tail, as defined in Sect. 6.

high-frequency radio emission is confined to within $\sim 10^{\prime \prime}$ of the active nucleus. The high-frequency observations reveal distorted structure with two lobes bent through $90^{\circ}$ within $\sim 2^{\prime \prime}$ of the nucleus.

The northern lobe appears more affected by the movement of the core. Figure 5 shows that the $\mathrm{N}$ lobe is well confined by an arc-shaped structure with temperature of $2.5 \mathrm{keV}$. This arc-like structure has been interpreted by Ehlert et al. (2015) as consistent with a cold front due to movement of the BCG northward. Similarly to the northern lobe, the southern lobe changes direction from SE to SW when it meets a region of higher pressure at around $1.5^{\prime \prime}$ to the SE of the active nucleus. After its deflection, the southern radio lobe flows through a channel of reduced pressure, which leads to the western part of the tail (see Sect. 6). The channel of lower pressure navigates the lobe toward the SW and allows it to expand in this direction.

\subsection{GMRT 610 and $235 \mathrm{MHz}$}

The radio map at $610 \mathrm{MHz}$ (Fig. 3) shows a round, not wellresolved morphology at the core of $\mathrm{A} 1795$. The $610 \mathrm{MHz}$ image also reveals the tail of a nearby background source at $z_{\text {phot }}=0.57 \pm 0.08$ (Giacintucci et al. 2014) situated to the west of the the central galaxy of A1795. The $235 \mathrm{MHz}$ map shows a smooth morphology close to the center of A1795 with a few marginally resolved extensions toward the NE, E, and S. The southern extension covers the western part of the X-ray tail (Fig. 6). We study this correspondence in Sect. 6. The most pronounced features in the $235 \mathrm{MHz}$ GMRT map are two clear extensions directed to the west of the active core. These extensions are significant at a $5 \sigma$ level. The radio emission of the northern extension spans up to $\sim 45 \mathrm{kpc}$ from the center, thus reaching beyond the NW edge, in the region of the NW X-ray depression (Fig. 6). The southern feature extends up to $\sim 50 \mathrm{kpc}$ and is associated with the SW X-ray depression.

We explore the correspondence between the $235 \mathrm{MHz}$ radio extensions and the X-ray depressions in Sect. 5.

\subsection{VLA 1.4 GHz C-configuration}

With the help of $1.4 \mathrm{GHz}$ VLA observations in $C$-configuration, Giacintucci et al. (2014) have studied the more extended radio structure of A1795 (Fig. 6). They find a candidate mini-halo extending up to a radius of $\sim 100 \mathrm{kpc}$ and measure a power for the mini-halo of $(7.9 \pm 0.5) \times 10^{23} \mathrm{~W} \mathrm{~Hz}^{-1}$. An important consequence of the map of Giacintucci et al. (2014) is that the NW depression is not covered by radio emission. Moreover, the highfrequency radio emission seems to surround the X-ray cavity, instead of filling it. Thus, the inner edge of the northern cavity resembles the southern "bay" feature in Perseus (Fabian et al. 2011). This is one of the factors that have made Walker et al. (2017) speculate that the NW X-ray concave enhancement is not a border of a cavity but a bay, similar to the one observed in 

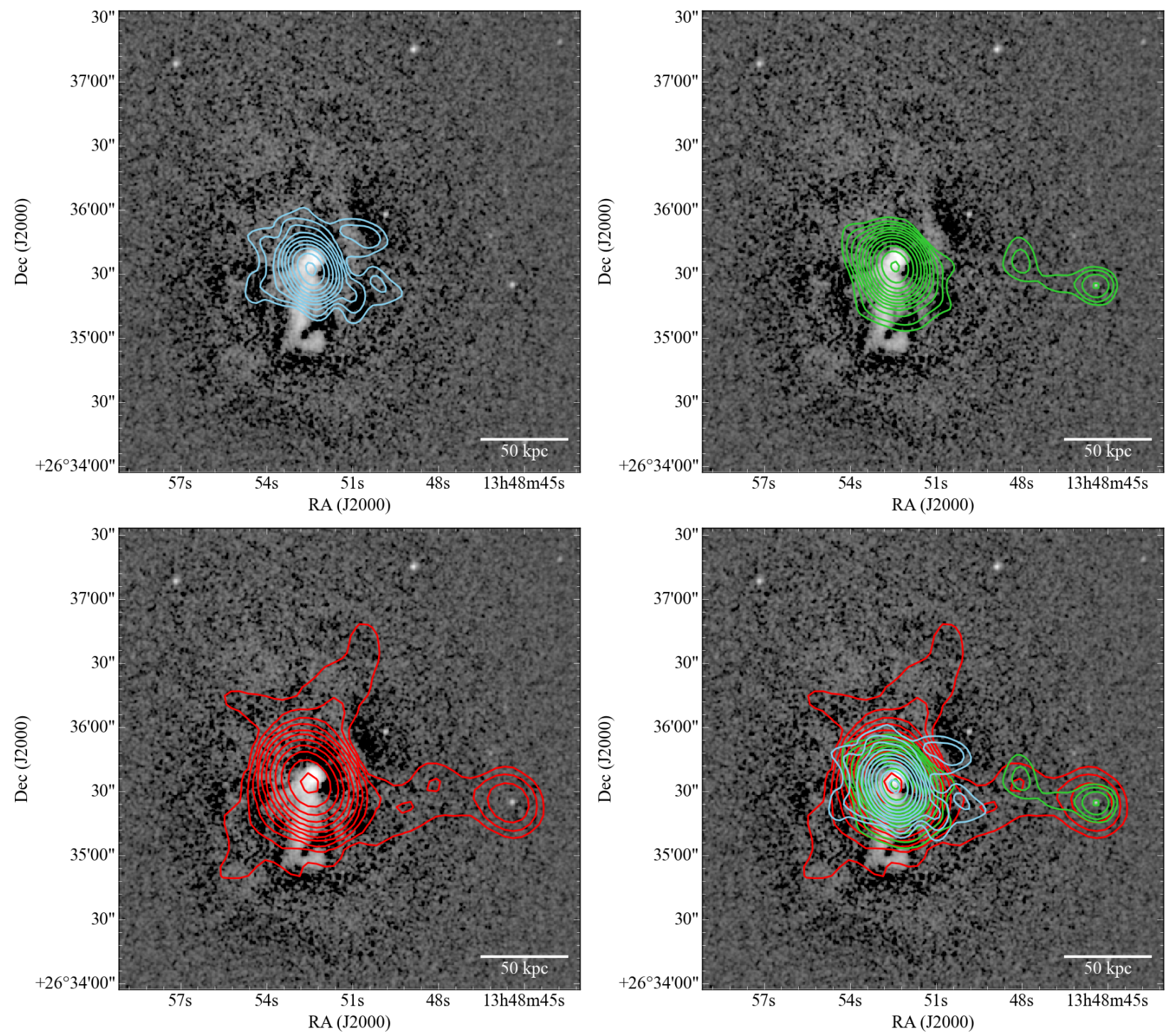

Fig. 6. X-ray residual map of A1795 with radio contours from different radio frequencies. Top left: contours from the GMRT image at $235 \mathrm{MHz}$. Top right: contours from the GMRT image at $610 \mathrm{MHz}$. The contour levels at 610 and $235 \mathrm{MHz}$ are the same as in Fig. 3. Bottom left: contours at $1.4 \mathrm{GHz}$ derived from the VLA $C$-configuration image of Giacintucci et al. (2014) are shown in red. They are drawn at $5 \sigma \times[1,2,4,8,16,32$, $64,128,256,512,1024,2048]$, where the rms noise level is $0.15 \mathrm{mJy}_{\text {beam }}^{-1}$. Bottom right: composite depicting the radio contours at $1.4 \mathrm{GHz}$ $C$-configuration (red), $610 \mathrm{MHz}$ (green), and $235 \mathrm{MHz}$ (blue).

Perseus. They further show that this bay may have been created by a Kelvin-Helmholtz $(\mathrm{KH})$ instability in the cold front surrounding the core of $\mathrm{A} 1795$.

The radio emission that appears to surround the NW X-ray cavity consists of two features, a radio filament toward the NW and a bridge of emission between the core and a source to the west. Although the NW extension in the $1.4 \mathrm{GHz}$ map of Giacintucci et al. (2014) is formally detected at the $5 \sigma$ significance level, it is possibly related to calibration uncertainty since it lies along one of the stripes of the typical VLA array pattern revealed in many images and resulting from the Y-shaped arrangement of the VLA antennas. If this feature were interpreted as being part of the mini-halo, then we would expect it to consist of older emission and therefore be more pronounced at lower frequencies. However, this extension does not appear in the GMRT maps, which supports our suspicion that the NW extension in the $1.4 \mathrm{GHz}$ map is significantly enhanced by a calibration artifact. Furthermore, we show that the bridge of radio emission between the central galaxy and the background source to the west is likely due to chance alignment (Fig. 6). Our GMRT map at $610 \mathrm{MHz}$ reveals that the background source has a wide tail of emission, with the plume of emission pointed toward the core of A1795. On the other hand, the $235 \mathrm{MHz}$ image shows an extension in the radio emission of A1795 stretching toward the SW. The simplest explanation is that the combination of these two features at lower resolution is what gives the appearance of a bridge of emission in the $1.4 \mathrm{GHz} C$-configuration map.

\section{Sector analysis}

Based on the discussion on the X-ray and radio morphology in the previous two sections we defined three wedge-shaped regions of interest centered on the BCG. To support our analysis, we extracted radial profiles confining regions along these major directions (Fig. 7). The NW profile follows the NW cavity and the northern radio extension at $235 \mathrm{MHz}$, while the SW wedge stretches along the SW cavity and the southern radio extension. The cold tail is covered by the southern profile. In 


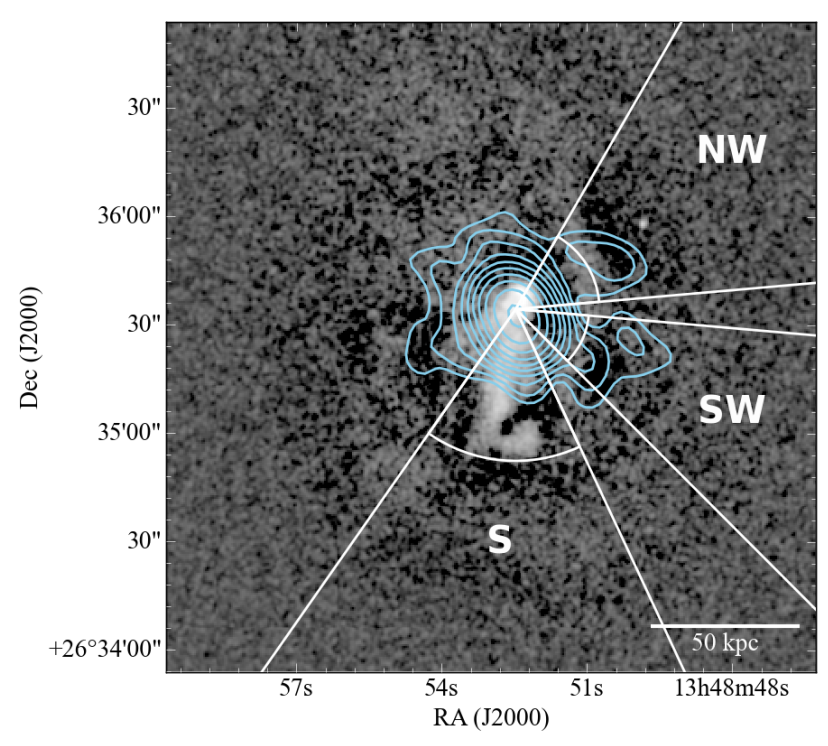

Fig. 7. Three sectors used for radial analysis extraction overplotted on the X-ray residual map from Fig. 2. The radio contours at $235 \mathrm{MHz}$ are shown in blue with the same levels as in Fig. 3. Arcs in the NW, SW, and S sector show respectively the positions of the NW edge, the SW edge, and the end of the tail as determined from the X-ray residual map.

addition we extracted an averaged radial profile in all directions. We present surface brightness, temperature, and metallicity profiles extracted from the X-ray data (Figs. 8 and 9), as well as radio surface brightness profiles at $235 \mathrm{MHz}$ (Fig. 10).

To construct the X-ray profiles the size of the bins in the radial direction is adapted so that each bin reaches a SN of 100 The radial extent of each bin in the radio profiles corresponds to the FWHM of the synthesized beam of the $235 \mathrm{MHz}$ image (shown in Fig. 3). The associated error is given by $\sigma_{\mathrm{n}} \times \sqrt{N_{\mathrm{b}}}$ where $\sigma_{\mathrm{n}}$ is the measured rms noise in the image and $N_{\mathrm{b}}$ is the number of beams sampled in each bin.

In this section we discuss the profiles in the NW and SW directions. The profile in the $S$ is analyzed in Sect. 6 where we study the properties of the cold tail.

\subsection{NW sector}

Three sectors used for radial analysis extraction overplotted on the X-ray residual map from Fig. 2. The radio contours at $235 \mathrm{MHz}$ are shown in blue with the same levels as in Fig. 3. Arcs in the NW, SW, and S sector show respectively the positions of the NW edge, the SW edge, and the end of the tail as determined from the X-ray residual map. Figure 8 shows the surface brightness profile along the NW sector. In the top left panel the NW profile is compared with the average profile. This comparison suggests two regions where the NW profile is much lower than the average value. To highlight the difference between the two profiles we have plotted the ratio of the NW to the average profile in the top right panel of Fig. 8. This ratio profile shows two pronounced dips (cavity-like structures) at the two sides of the NW edge. These regions correspond to the NW and the arc depressions identified in the residual map.

The ratio of the temperature profiles (Fig. 8) shows two temperature enhancements corresponding to the two dips in the $\mathrm{X}$-ray surface brightness. While the surface brightness dips might be suggestive of shock fronts or cold fronts due to sloshing, the temperature profile does not support these possibilities. The temperature profiles do not exhibit any sharp jumps or drops, but rather a smooth increase in temperature peaking at the mid-length of the surface brightness depressions. This temperature behavior is more consistent with a cavity structure produced by the AGN injecting hot plasma within the bubbles.

Based on the ratio of the X-ray surface brightness profiles, the outer depression is situated $44 \mathrm{kpc}$ from the center and has a radius of $10 \mathrm{kpc}$, assuming spherical symmetry. This corresponds to an age of $38 \mathrm{Myr}$, based on the sound speed in this region. Assuming the cavity is filled with relativistic plasma, its total energy is $E=4 p V=4 \times 10^{59} \mathrm{erg}$ and the power of the outburst is estimated to be $7 \times 10^{44} \mathrm{erg} \mathrm{s}^{-1}$.

While the NW cavity is one of the most distant cavities observed, the measured energy and power are close to the average values known from other systems. We note that the total energy we compute is an order or magnitude lower than the value presented by Walker et al. (2014). This follows from the size they infer from the curvature of the inner rim of the cavity, which is almost two times larger. The difference in size of the cavity also contributes to the longer sound crossing time (41 Myr) they measure.

The arc depression has a radius of $7 \mathrm{kpc}$. It is located at a distance $16 \mathrm{kpc}$ from the center, which corresponds to an age of $14 \mathrm{Myr}$. Since this cavity is clearly non-spherical, we estimate its volume based on a part of a torus with a length of $23 \mathrm{kpc}$ derived from the residual map. We compute a total energy of $5 \times 10^{58} \mathrm{erg}$ and infer a power of the AGN outburst of $9 \times 10^{43} \mathrm{erg} \mathrm{s}^{-1}$. If the NW depression and the arc depression indeed resulted from the AGN activity, then the outbursts that created them have almost an order of magnitude difference in power.

The top panel of Fig. 10 presents the radial profile extracted from the $235 \mathrm{MHz}$ map along the NW radio extension. The plotted profile shows clearly extended radio emission in the NW direction. The statistically significant emission at $235 \mathrm{MHz}$ reaches beyond the NW edge, up to $\sim 40 \mathrm{kpc}$ from the center, and covers the inner part of the NW cavity. As already seen from the maps in Fig. 6, the arc cavity is fully covered by low-frequency radio emission. The fact that we observe radio emission corresponding to the NW and the arc depressions provides another piece of strong evidence that the observed X-ray depressions are the result of previous AGN activity. We note that it is hard to draw a definitive conclusion about the origin of the arc cavity because is shows an unusual shape and in general the ICM morphology is very complex in this direction. However, the radio source is clearly disturbing and distorting the medium in that direction which is consistent with the cavity interpretation for the arc depression.

\subsection{SW sector}

The SW profiles shown in Fig. 9 allow us to identify two regions of decreased X-ray surface brightness. They are both located beyond the SW edge, at distances of 33 and $78 \mathrm{kpc}$ from the central SMBH. Although the SW sector passes over the arc cavity (Fig. 7), it is difficult to trace this cavity in the surface brightness profile. This is likely due to the insufficient number of bins within the inner $10 \mathrm{kpc}$ from the SMBH which would help us discern the inner edge of the cavity.

The inner depression distinguished in our surface brightness profile corresponds to the SW depression identified in Sect. 3. We observe a significant temperature enhancement corresponding to this depression (Fig. 9) which supports its classification 
G. Kokotanekov et al.: Signatures of multiple episodes of AGN activity in the core of Abell 1795
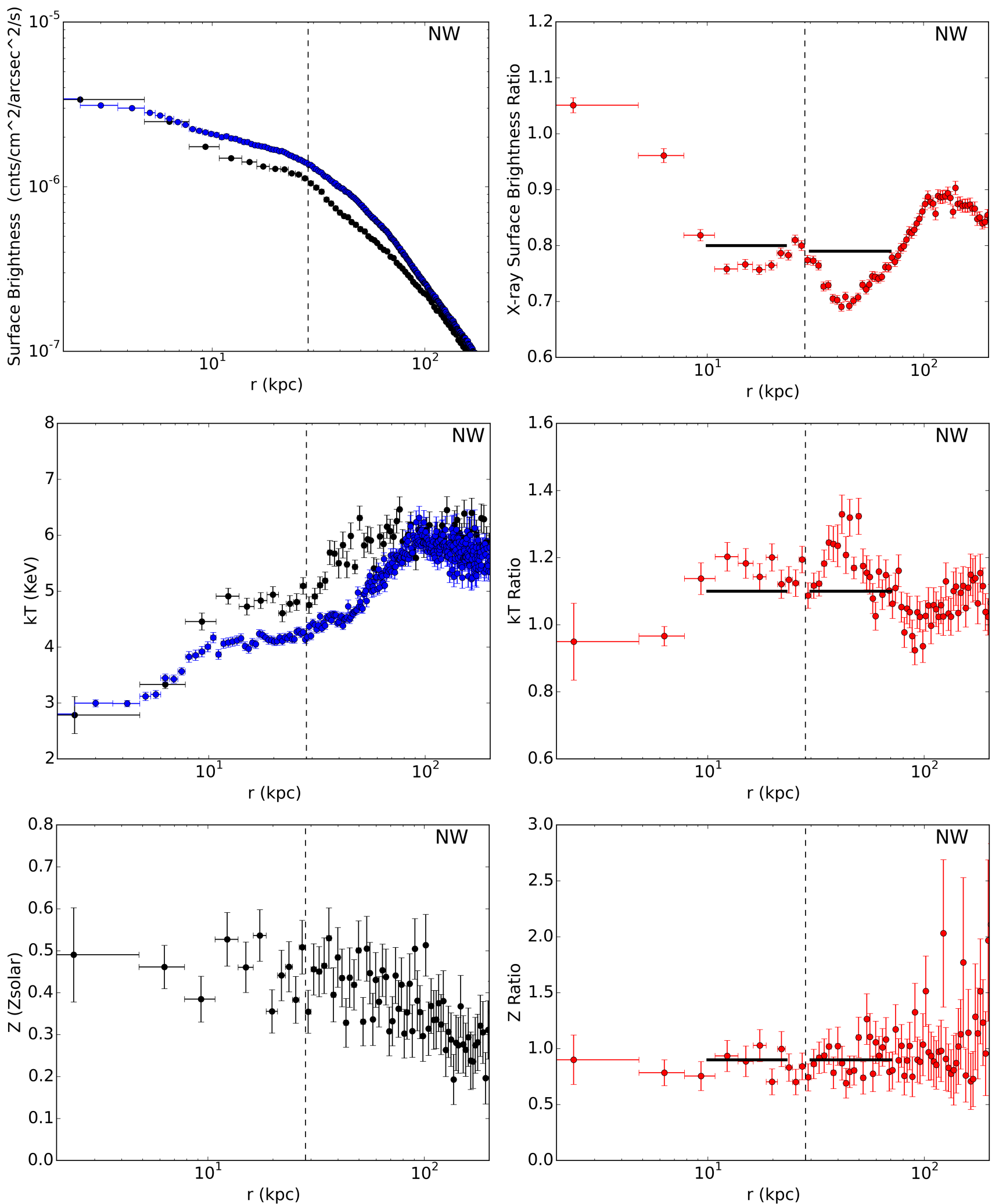

Fig. 8. X-ray radial profiles along the NW direction. Top left: surface brightness profile along the NW (black) and average surface brightness profile (blue). Top right: ratio of the X-ray surface brightness profile toward the NW to the average X-ray surface brightness profile. Middle left: temperature profile along the NW (black) and average temperature profile (blue). Middle right: ratio of the temperature profile toward the NW to the average temperature profile. Bottom left: metallicity profile along the NW. Bottom right: ratio of the metallicity profile toward the NW to the average metallicity profile. The average metallicity profile can be seen in Fig. 12. The black stripes show the extent of the depressions as constrained from the X-ray surface brightness ratio profile. The vertical dashed line indicates the position of the NW edge as determined from the X-ray residual map (Fig. 7). 

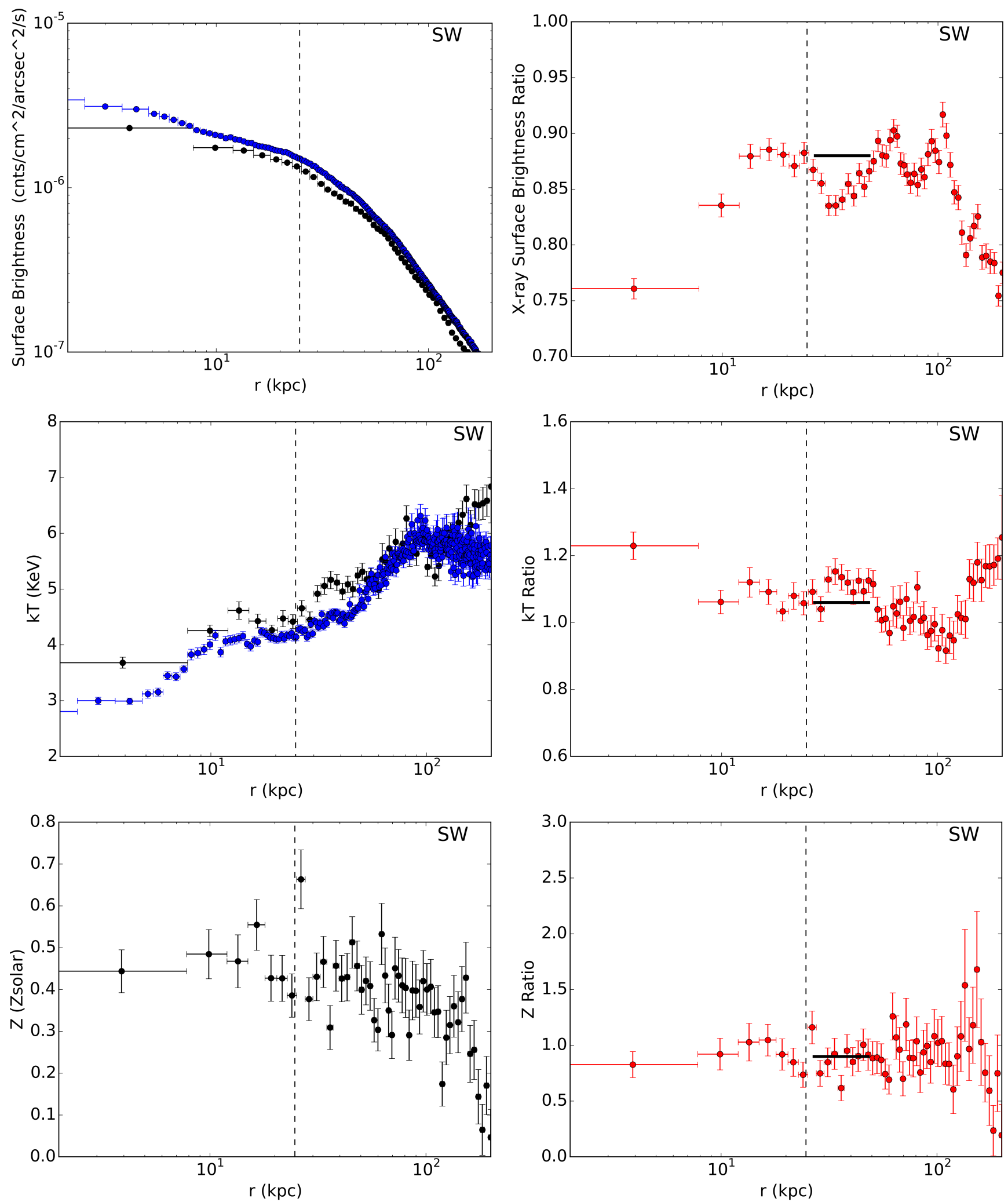

Fig. 9. X-ray radial profiles along the SW direction. Top left: surface brightness profile along the SW (black) and average surface brightness profile (blue). Top right: ratio of the X-ray surface brightness profile toward the SW to the average X-ray surface brightness profile. Middle left: temperature profile along the SW (black) and average temperature profile (blue). Middle right: ratio of the temperature profile toward the SW to the average temperature profile. Bottom left: metallicity profile along the SW. Bottom right: ratio of the metallicity profile toward the SW to the average metallicity profile. The average metallicity profile can be seen in Fig. 12. The black stripe shows the extent of the SW depression as constrained from the X-ray surface brightness ratio profile. The vertical blue line shows the position of the SW edge as determined from the X-ray residual map (Fig. 7). 

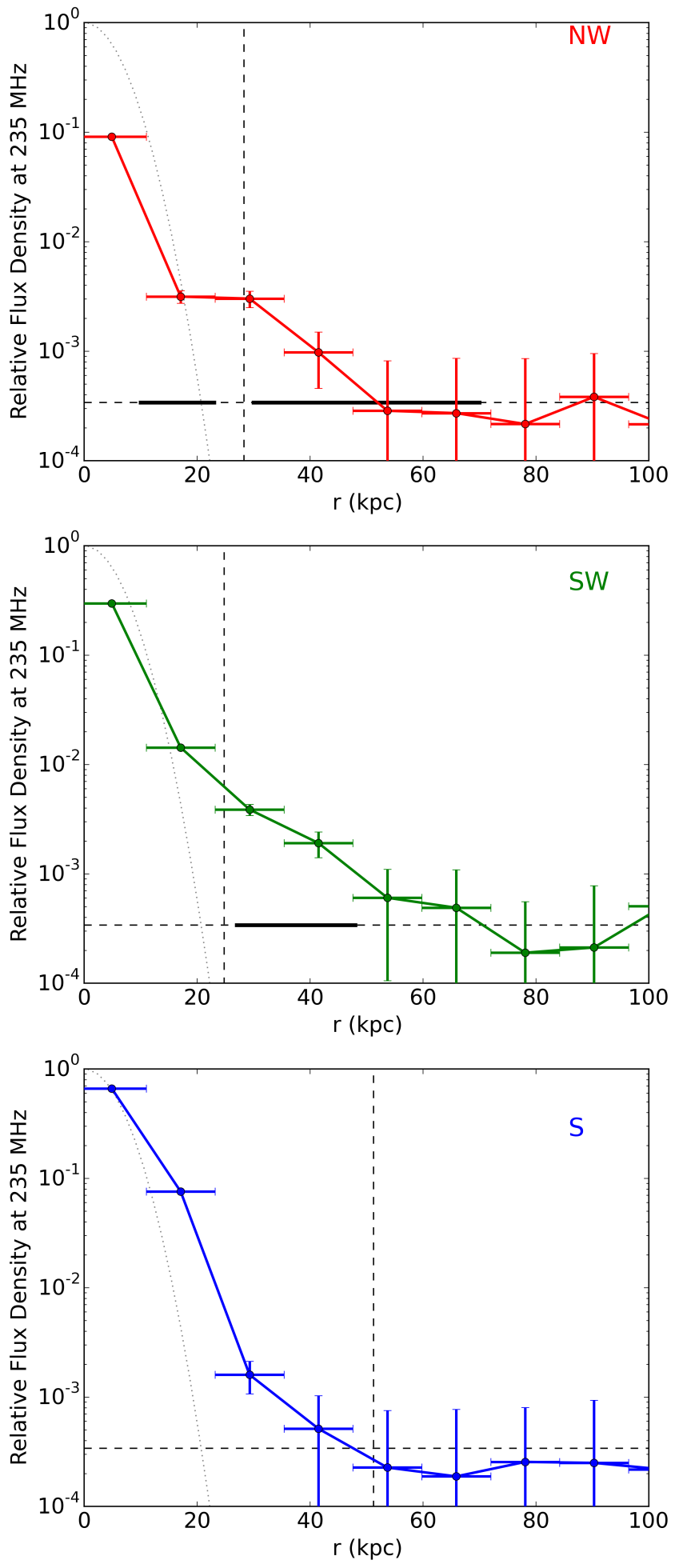

Fig. 10. Radial radio profiles at $235 \mathrm{MHz}$ in the NW (top panel), SW (middle panel), and S (bottom panel) directions. The vertical lines give the positions of the NW edge, SW edge, and the end of the S tail, as determined from the X-ray residual map (Fig. 7). The horizontal dashed line indicates the rms noise level in the image. The black stripes show the extent of the depressions as constrained from the X-ray surface brightness ratio profiles (Figs. 8 and 9). A Gaussian characteristic of the beam is shown with a gray dotted line to emphasize that the observed extended radio structures are significant in comparison with the beam size. The flux density is presented in relative units with respect to the peak flux measured at the center of A1795. as a cavity. The observed temperature behavior suggests that the observed depression contains hotter plasma presumably injected by the AGN. An alternative explanation is that the temperature appears to rise within the cavities simply because the cavity has displaced cooler X-ray gas, thus hotter material dominates along the line of sight. We compute an age of $30 \mathrm{Myr}$ for the SW cavity, assuming propagation with the local sound speed. The total energy of the cavity is $7 \times 10^{58} \mathrm{erg}$, while the cavity power is $1 \times 10^{44} \mathrm{erg} \mathrm{s}^{-1}$.

The radio profile along the SW sector resembles the profile in the NW direction (Fig. 10). While the bulk of the emission is clearly restricted by the edges in both the NW and SW, the radio plasma is not fully contained by them. Similar to the picture observed toward the NW, the SW radio profile exhibits an extension spanning up to $\sim 50 \mathrm{kpc}$ from the center. The radio plasma toward the SW appears more extended than in the NW and it fully fills in the SW cavity. This configuration additionally confirms that this cavity is a product of a past AGN activity episode.

Despite our interpretation of the cavities along the NW and SW directions as the products of past AGN activity, we note that the measured abundance profiles are relatively flat over the central $100 \mathrm{kpc}$ and show no evidence of significant extensions of enhanced metallicity along these directions relative to the average profile (Figs. 8 and 9). At first glance, this result would seem to contradict the results of Kirkpatrick \& McNamara (2015) who have found evidence of a metallicity extension along the axis of the radio jet in the core of A1795. The "on-jet" regions defined in Kirkpatrick \& McNamara (2015), however, only overlap with our S sector containing the S cold tail. For their "off-jet" direction, defined to be orthogonal to the on-jet direction and more closely aligned with our NW and SW sectors, they also found the region of enhanced metallicity in the core to be less extended. If the proposed NW and SW cavities are indeed relics of past AGN activity, they do not seem to have lifted metal-enriched gas to larger radii to the extent seen by Kirkpatrick \& McNamara (2015) in the $\mathrm{N}$ and $\mathrm{S}$ directions.

The outer dip in our surface brightness radial profile (Fig. 9) seems to be part of a large-scale spiral structure of low intensity surrounding the core. It is evident from Fig. 7 that only a small part of this spiral feature falls within the SW sector. The elongated structure, which seems to stretch from the end of the tail up to the northern end of the SW sector, does not morphologically resemble a cavity, but rather suggests a shock or a cold front. Moreover, we do not distinguish a temperature bump associated with the outer surface brightness dip. We only find a drop in temperature, which is more consistent with a cold front. Based on this we conclude that the outer depression in the X-ray surface brightness profile does not correspond to an AGN outflow cavity.

\subsection{Spectral index profiles}

After matching the resolutions of the images at 235 and $610 \mathrm{MHz}$, we have extracted radial profiles from the two frequencies and used them to reconstruct a spectral index profile along the three chosen directions (Fig. 11). The obtained spectral index profiles reveal very different behavior of the plasma in the NW and SW compared to the S. The spectral index of the radio emission between 235 and $610 \mathrm{MHz}$ remains relatively flat in the $\mathrm{S}$ direction. It changes from -0.7 close to the center to -1.0 at the tail. Similarly, the spectral index in the NW and SW stays flatter than -1.0 inside the NW and SW edges. Outside 


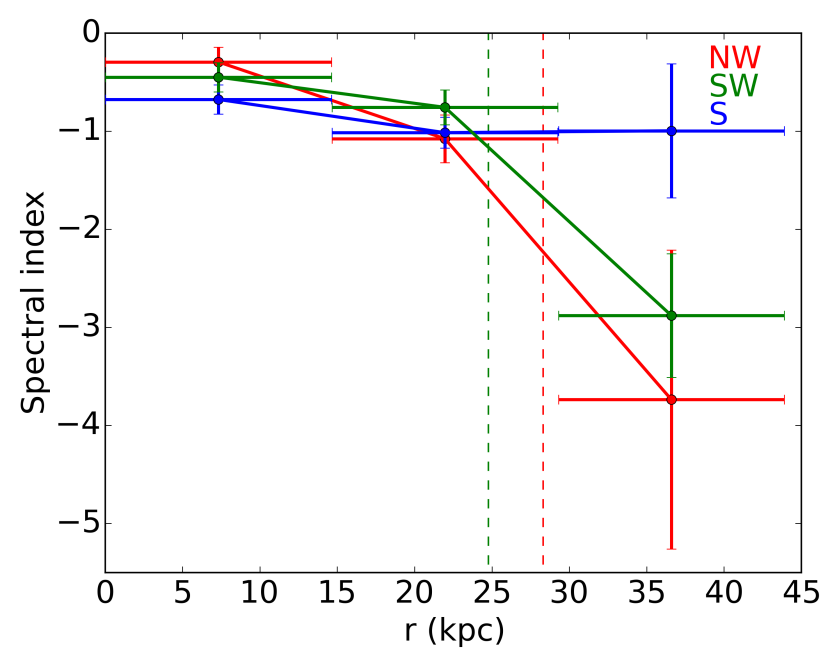

Fig. 11. Spectral index between $610 \mathrm{MHz}$ and $235 \mathrm{MHz}$. The vertical lines show the positions of the NW (red) and the SW (green) edges. In addition to the statistical errors, the errors of the spectral index include $10 \%$ uncertainty in the flux scale of the two frequencies used.

the edges, however, the emission in these two directions appears very aged exhibiting a spectral index lower than -2.9 . The spectral index profiles in radio are consistent with the interpretation that extensions observed in the GMRT map at $235 \mathrm{MHz}$ consist of aged plasma, produced from an older outburst. The similar spectral properties of the two extensions suggest that they have similar ages and are thus likely produced by the same AGN outburst.

\section{Cold tail}

The southern tail feature has been studied extensively and a number of explanations have been proposed for its origin. One possibility is that the tail resulted from an outflow of material triggered by the central SMBH. An alternative interpretation states that the giant filament consists of cold gas from the central galaxy that has been stripped out by dynamical friction. Fabian et al. (2001) suggest that the cold tail is due to a "cooling wake" produced as the central cluster galaxy oscillates through the core of the cluster. In this model the X-ray bright ICM cooled around the motion of the BCG, which also gave rise to an $\mathrm{H} \alpha$ filament (Fabian et al. 2001; McDonald \& Veilleux 2009). Markevitch et al. (2001) argue that the BCG stays with the gravitational potential to within its observed small $150 \mathrm{~km} \mathrm{~s}^{-1}$ velocity relative to the cluster average (Oegerle \& Hill 1994), while the relative motion between the $\mathrm{BCG}$ and the gas results from the sloshing of the gas.

The AGN origin has been seen as unlikely since no compelling signatures of AGN activity, such as X-ray cavities or radio emission, have been found to accompany the tail in its full extent. While the stripping option requires a complicated scenario that most likely involves ram pressure stripping beyond the scales of the BCG (see Ehlert et al. 2015), the cooling wake model is supported by the bulk of the observational evidence on the tail's dynamics. The gas motions in this filament have been found to be modest and not turbulent (Crawford et al. 2005). Additionally, Hu et al. (1985) find that most of the optical filament that accompanies the X-ray tail shares the velocity of the cluster rather than that of the BCG. This velocity configuration agrees well with the cooling scenario, and argues against the stripping model where a significant velocity gradient is expected.

The radio emission in our map at $235 \mathrm{MHz}$ covers the inner parts of the tail up to $\sim 30 \mathrm{kpc}$ from the core. It is evident from the radial profiles at $235 \mathrm{MHz}$ (Fig. 10) that the radio emission in the $\mathrm{S}$ is not as extended as in the NW and SW. The lack of an extended, aged radio plasma structure clearly aligned with the main body of the X-ray tail argues against the possibility that the tail was created by the AGN activity. Similarly, our radio maps show that no low-frequency emission is associated with the hook feature and the small-scale X-ray surface brightness hole at the end of the tail discussed by Crawford et al. (2005) and Walker et al. (2014). The lack of corresponding low-frequency radio emission for both the hook feature and the small depression further disfavors an AGN origin for the cold tail.

The temperature profile along the tail (Fig. 12) confirms that south of the core the overall temperature is significantly lower than the average, but does not demonstrate a significant gradient along the direction of the tail. A jump in temperature is only evident at the end of the tail at $\sim 45 \mathrm{kpc}$, after which the temperature increases rapidly. The lack of a significant temperature gradient along the tail suggests that there is little or no mixing between the gas in the tail and the surrounding ICM. It also shows that there is no significant cooling by the gas in the tail over the time interval required for the tail to reach its current length.

Using the available temperature and pressure information, we have derived the cooling properties of the gas in the tail. We find no significant gradient in the cooling time along the tail and measure an average cooling time of $500 \pm 70 \mathrm{Myr}$ in a region encompassing the tail. This average is consistent with the age of $\sim 300$ Myr we compute for the end of the tail using the velocity $+150 \mathrm{~km} \mathrm{~s}^{-1}$ as measured by Oegerle \& Hill (1994). We note that obtaining the cooling time requires making assumptions about the geometry of the regions in order to derive the reprojected gas densities. These assumptions introduce a systematic uncertainty that is potentially larger than the quoted statistical uncertainty, but would not change the qualitative agreement between the two age estimates. Furthermore, we detect no strong metallicity gradient in the tail (Fig. 12), which might be expected if the tail consists of material that has been dragged from the central BCG. Thus, all the presented evidence argues in favor of the cooling wake hypothesis.

Based on the X-ray residual and temperature maps, the tail can be divided into two main parts, an inner round part and an outer straight part (Figs. 5 and 13). The inner tail is separated from the outer tail by a bridge of warmer $(3.5 \mathrm{keV})$ material situated at $\sim 6 \mathrm{kpc}$ from the radio core. The inner tail is situated around the active core, it is not well confined, and is elongated toward the SE. The outer part of the tail appears quite linear, but exhibits an inhomogeneous and knotty structure on smaller scales. The outer tail extends to the $S$ out to a distance of $47 \mathrm{kpc}$ from the center.

Our interpretation of the different morphology of the inner and outer tail is that we are looking at a superposition of two major phenomena. One is the dynamical movement of the central galaxy, which results in a cooling wake, and the other is related to the most recent episodes of AGN, which drives material out. While the outer tail is governed by the movement of the BCG, the inner tail seems to be significantly affected by the AGN activity. The inner tail follows the apparent NW-SE symmetry axis of enhanced pressure, presumably tracing the older AGN outflows. We suggest that the AGN activity has likely disrupted the 

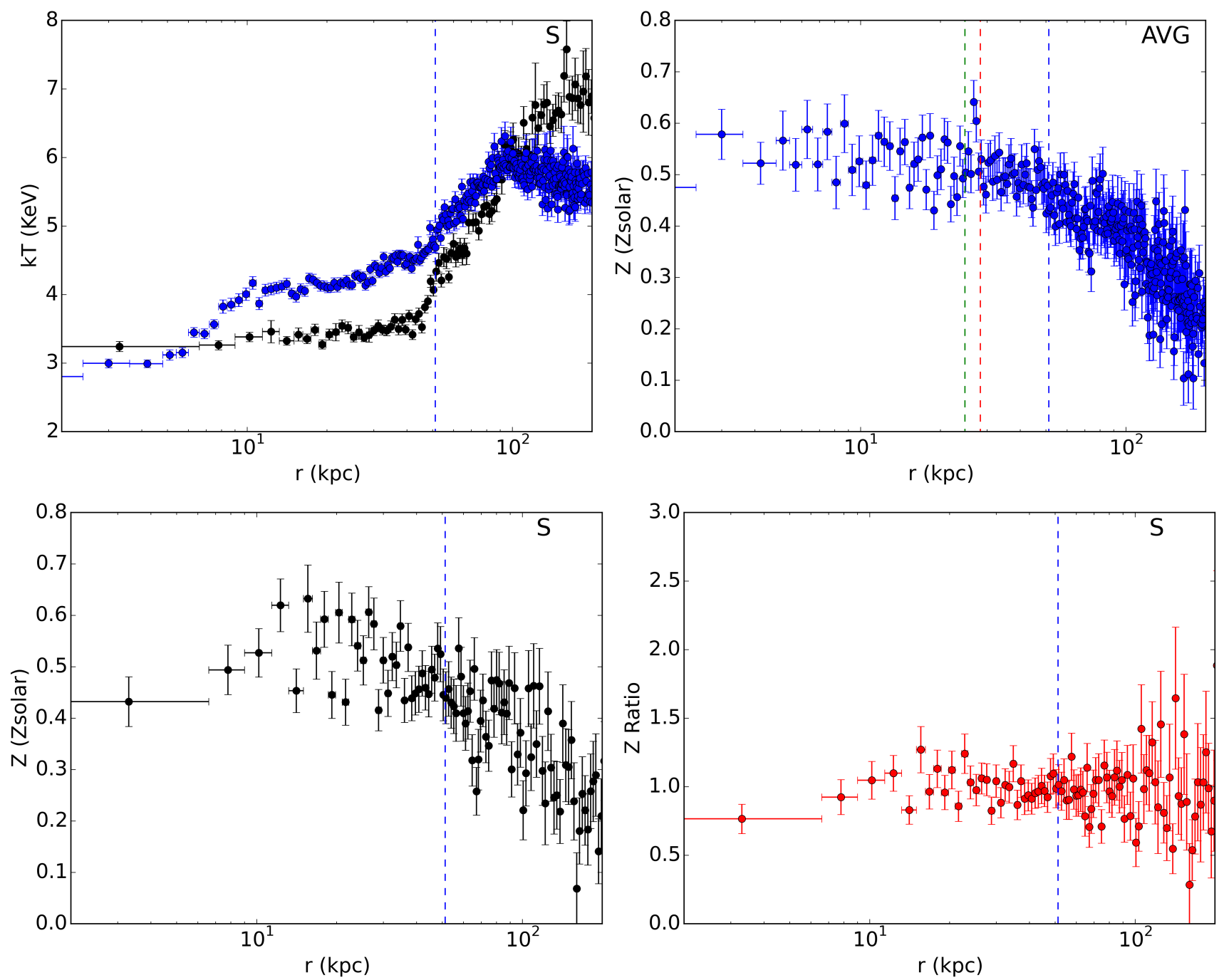

Fig. 12. Top left: temperature radial profile along the $S$ direction (black) and average temperature profile (blue). Top right: average metallicity profile. Bottom left: metallicity profile along the S. Bottom right: ratio of the metallicity profile toward the $\mathrm{S}$ to the average metallicity profile. The vertical blue line indicates the end of the tail as determined from the X-ray residual map (Fig. 7). The red and green vertical lines in the average metallicity plot indicate the position of the NW and SW edges, respectively.

cooling wake, introducing the observed gap between the inner and outer tail, as well as the irregular shape of the inner tail and the kinks in the outer tail closer to the center.

\section{Discussion and conclusions}

When revealed through shallow X-ray and high-frequency radio data, the morphology at the cores of relaxed cool-core clusters often looks simple and symmetric. However, studying these objects with deeper and multiwavelength observations we find many complicated processes all acting in the same place. In this work we presented extremely deep Chandra X-ray data combined with GMRT radio observations at $235 \mathrm{MHz}$ and $610 \mathrm{MHz}$. We study A1795 as a dramatic example of a cluster core with rich X-ray morphology. In this source the distinguished features are not all linked to a common origin. In A1795 we are seeing motion of the core but also activity from the AGN. These two phenomena act together to shape a very complicated picture, which makes it difficult to distinguish their individual effects.

Using a spatially resolved, X-ray spectral analysis, we identify the known X-ray depression in the NW direction and determine a distance of $44 \mathrm{kpc}$ from the AGN. The inner rim of this depression is a bright edge of $\mathrm{X}$-ray emission, situated $28 \mathrm{kpc}$ from the core. Due to its temperature structure, this elongated structure is consistent with a cold front. Although no observed radio emission has previously been associated with the NW depression, our observation at $235 \mathrm{MHz}$ shows that an extended filament of radio plasma connects the NW depression with the active core. This configuration provides strong circumstantial evidence that the surface brightness depression is a morphological signature of past AGN activity, and thus supports the classification of this depression as a cavity. Based on the local sound speed we measure an age of $38 \mathrm{Myr}$ for the cavity. The power of the corresponding outburst is estimated to be $7 \times 10^{44} \mathrm{erg} \mathrm{s}^{-1}$.

The deep Chandra data allowed us to identify two other cavities, a SW cavity and an arc cavity. Similarly to the case in the $\mathrm{NW}$, in the SW direction we identified an edge (situated $25 \mathrm{kpc}$ from the center) and an outer cavity fully covered by an extension of the radio plasma at $235 \mathrm{MHz}$. For the SW cavity we computed an age of $30 \mathrm{Myr}$ and a power of $1 \times 10^{44} \mathrm{erg} \mathrm{s}^{-1}$. Closer to the core we observed a very elongated arc-shaped cavity. It is situated outside of the arc-like inner edge and is separated from the NW and the SW cavities by the NW and SW edges, respectively. The arc cavity is located at a distance of 


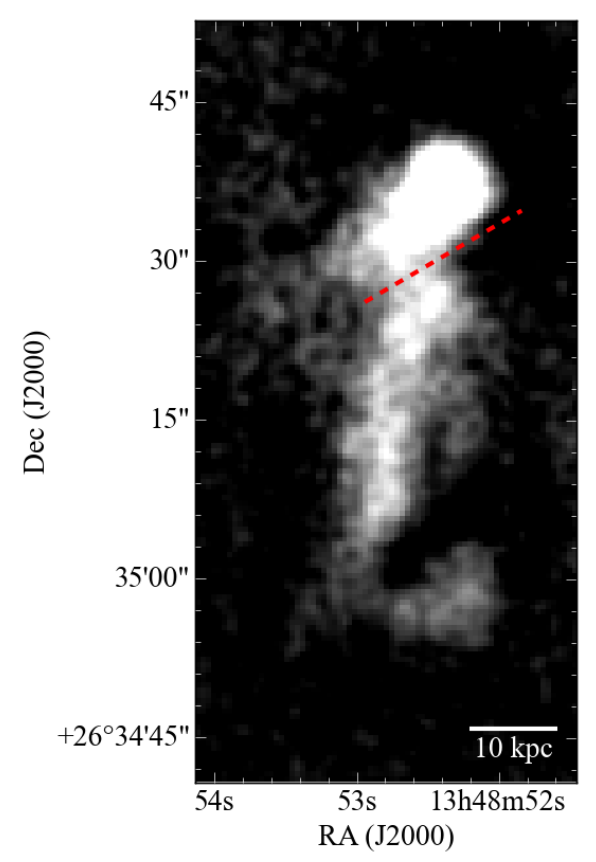

Fig. 13. Section of the X-ray residual map showing the cold tail. The dashed line shows the division between inner and outer tail as defined in the text.

$16 \mathrm{kpc} \mathrm{NW}$ from the center, which corresponds to an age of 14 Myr. We compute a corresponding power of the AGN outburst of $9 \times 10^{43} \mathrm{erg} \mathrm{s}^{-1}$. All three identified cavity structures are clearly distinguished as depressions in the radial surface brightness profiles and also align with peaks of hotter emission in the extracted temperature profiles. While the cavities appear to be filled with hotter plasma, presumably injected by the AGN, they do not seem to have dredged up enhanced metallicity gas.

We interpret the complicated structure seen in the NW direction as the signature of three epochs of AGN activity. We see two edges: the NW edge and the arc edge, which is closer to the core. Those two regions of compressed material serve as the boundaries between the three cavities corresponding to the three outbursts. The oldest is the NW cavity, then followed by the arc cavity. Inside the arc edge there are inner cavities, identified by Ehlert et al. (2015), that are currently being inflated by the radio jets. A similar picture is revealed in the SW; however, the signatures of AGN activity in this direction are less pronounced, most likely due to the effects of the moving core and the cooling wake. Beyond the SW edge we observe the outer SW cavity, while closer to the core is the southern part of the arc cavity. The elongated shape and the location of the arc cavity, shared between the NW and SW directions, may suggest that this irregular cavity is a result of the merger of two cavities produced by the same outburst.

The observed radio behavior further supports the severalburst model. The emission corresponding to the two radio extensions reaching beyond the edges appears very aged, exhibiting a spectral index between 235 and $610 \mathrm{MHz}$ steeper than -2.9 . The spectral index profiles in radio support the notion that the extensions observed in the GMRT map at $235 \mathrm{MHz}$ consist of aged plasma, presumably produced from an older outburst. Moreover, the similar spectral properties of the two extensions suggest that they have similar ages and are thus likely produced by the same AGN outburst.

On the other hand, the spectral index of the radio emission remains relatively flat (flatter than -1.0 ) inside the two edges.
In this region, the new activity appears to be offsetting the ongoing spectral aging process. The reacceleration process flattens the spectrum inside the edge, while the plasma outside of the edge is fully decoupled from the AGN and continues to age.

The new deep X-ray data allows us to separate the $\sim 47 \mathrm{kpc}$ tail into two main parts with very different structures. While the inner part is round, the outer tail appears straight and very confined. Based on the different morphology at the two parts of the tail, we suggest that the outer tail is governed by the movement of the BCG, while the inner tail is severely disturbed by the AGN activity. In this scenario, the observed gap between the inner and outer tail, the irregular shape of the inner tail, and the kinks observed in the outer tail are all attributed to the activity of the AGN.

Even at $235 \mathrm{MHz}$ we do not observe radio emission corresponding to the extended cold tail. The lack of this emission disfavors an AGN origin of the tail. Furthermore, despite the depth of the X-ray data, we detect no significant temperature, cooling time, or metallicity gradient along the tail. We measure an average cooling time of $500 \pm 70 \mathrm{Myr}$, which is consistent with the age of $\sim 300$ Myr derived from the velocity of the core movement (Oegerle \& Hill 1994). All of the present evidence supports the cooling wake hypothesis for the origin of the tail.

Giacintucci et al. (2014) have identified a mini-halo in the system and determine its size and power based on a VLA observation taken with the $C$-array configuration. They estimate a size for the mini-halo of $\sim 100 \mathrm{kpc}$. Although the resolution of the GMRT images presented here is higher than the images of Giacintucci et al. (2014), we do not resolve the region of the mini-halo inside a radius of $\sim 20 \mathrm{kpc}$ from the radio core. However, beyond $\sim 30 \mathrm{kpc}$, we observe signatures of AGN activity which are consistent with the X-ray data and are supported by the spectral index derived between 235 and $610 \mathrm{MHz}$. Since our analysis interprets some of the extended radio emission to be a result of AGN activity, the implication is that the minihalo is smaller in size and power than originally inferred by Giacintucci et al. (2014).

In this work, we have presented evidence for the signatures of multiple episodes of AGN activity in the core of the A1795 cluster. Despite the use of very deep X-ray and broadband radio data, it is still difficult to disentangle the observed morphology of the core into a simple feedback-driven model. The ongoing AGN activity is clearly superimposed on core structures related to the motion of the central BCG, the apparent sloshing of the ICM gas in the central potential, and the formation of the cooling wake these motions have induced. In the radio, the observed morphology is similarly complicated, due to the presence of what appears to be aged relic emission from past AGN activity mingled with an apparent radio mini-halo in the core. It is possible that we are looking at a mini-halo being formed by multiple episodes of activity and the complicated picture revealed in the core of A1795 may ultimately provide evidence of the mechanism responsible for building mini-halos. Future higher resolution, low-frequency radio data from LOFAR and gas velocity information provided by future missions such as Athena may allow us to separate these overlapping physical effects and better constrain the evolution of the observed radio and X-ray structures in the core of A1795 over time.

Acknowledgements. We thank Laura Bîrzan and Simona Giacintucci for kindly providing their VLA images at $1.4 \mathrm{GHz}$. GDK acknowledges support from NOVA (Nederlandse Onderzoekschool voor Astronomie). GMRT is run by the National Centre for Radio Astrophysics of the Tata Institute of Fundamental Research. This research has made use of the NASA/IPAC Extragalactic Database (NED) which is operated by the Jet Propulsion Laboratory, California Institute 
of Technology, under contract with the National Aeronautics and Space Administration. We have also used SAOImage DS9, developed by Smithsonian Astrophysical Observatory.

\section{References}

Bîrzan, L., McNamara, B. R., Nulsen, P. E. J., Carilli, C. L., \& Wise, M. W. 2008 ApJ, 686, 859

Blanton, E. L., Randall, S. W., Clarke, T. E., et al. 2011, ApJ, 737, 99

Boehringer, H., Voges, W., Fabian, A. C., Edge, A. C., \& Neumann, D. M. 1993 MNRAS, 264, L25

Buote, D. A., \& Tsai, J. C. 1996, ApJ, 458, 27

Burns, J. O., Sulkanen, M. E., Gisler, G. R., \& Perley, R. A. 1992, ApJ, 388, L49

Cowie, L. L., Hu, E. M., Jenkins, E. B., \& York, D. G. 1983, ApJ, 272, 29

Crawford, C. S., Sanders, J. S., \& Fabian, A. C. 2005, MNRAS, 361, 17

de Gasperin, F., Orrú, E., Murgia, M., et al. 2012, A\&A, 547, A56

Ehlert, S., Allen, S. W., von der Linden, A., et al. 2011, MNRAS, 411, 164

Ehlert, S., McDonald, M., David, L. P., Miller, E. D., \& Bautz, M. W. 2015, ApJ, 799,174

Ettori, S., Fabian, A. C., Allen, S. W., \& Johnstone, R. M. 2002, MNRAS, 331, 635

Fabian, A. C., Sanders, J. S., Ettori, S., et al. 2000, MNRAS, 318, L65

Fabian, A. C., Sanders, J. S., Ettori, S., et al. 2001, MNRAS, 321, L33

Fabian, A. C., Sanders, J. S., Taylor, G. B., et al. 2006, MNRAS, 366, 417

Fabian, A. C., Sanders, J. S., Allen, S. W., et al. 2011, MNRAS, 418, 2154

Ferrarese, L., \& Merritt, D. 2000, ApJ, 539, L9

Fruscione, A., McDowell, J. C., Allen, G. E., et al. 2006, in Proc. SPIE, 6270, $62701 \mathrm{~V}$

Ge, J. P., \& Owen, F. N. 1993, AJ, 105, 778
Gebhardt, K., Bender, R., Bower, G., et al. 2000, ApJ, 539, L13

Giacintucci, S., Markevitch, M., Venturi, T., et al. 2014, ApJ, 781, 9

Hu, E. M., Cowie, L. L., \& Wang, Z. 1985, ApJS, 59, 447

Intema, H. T. 2014, ASI Conf. Ser., 13, 469

Intema, H. T., van der Tol, S., Cotton, W. D., et al. 2009, A\&A, 501, 1185

Intema, H. T., Jagannathan, P., Mooley, K. P., \& Frail, D. A. 2017, A\&A, 598, A78

Kalberla, P. M. W., Burton, W. B., Hartmann, D., et al. 2005, A\&A, 440, 775

Kirkpatrick, C. C., \& McNamara, B. R. 2015, MNRAS, 452, 4361

Kokotanekov, G., Wise, M., Heald, G. H., et al. 2017, A\&A, 605, A48

Markevitch, M., Vikhlinin, A., \& Mazzotta, P. 2001, ApJ, 562, L153

McDonald, M., \& Veilleux, S. 2009, ApJ, 703, L172

McNamara, B. R., Wise, M., Nulsen, P. E. J., et al. 2000, ApJ, 534, L135

Nulsen, P. E. J., David, L. P., McNamara, B. R., et al. 2002, ApJ, 568, 163

Oegerle, W. R., \& Hill, J. M. 1994, AJ, 107, 857

Radon, J. 1986, IEEE Trans. Med. Imaging, MI-5, 170

Sanders, J. S. 2006, MNRAS, 371, 829

Scaife, A. M. M., \& Heald, G. H. 2012, MNRAS, 423, L30

Sijbring, L. G. 1993, A Radio Continuum and HI Line Study of the Perseus Cluster

Silk, J., \& Rees, M. J. 1998, A\&A, 331, L1

Smith, R., Brickhouse, N., Liedahl, D., \& Raymond, J. 2001, ApJ, 556, L91

Swarup, G. 1991, in Radio Interferometry. Theory, Techniques, and Applications, 19, eds. T. J. Cornwell, \& R. A. Perley, ASP Conf. Ser., IAU Colloq., 131, 376

Walker, S. A., Fabian, A. C., \& Kosec, P. 2014, MNRAS, 445, 3444

Walker, S. A., Hlavacek-Larrondo, J., Gendron-Marsolais, M., et al. 2017, MNRAS, 468, 2506

Wise, M. W., McNamara, B. R., Nulsen, P. E. J., Houck, J. C., \& David, L. P. 2007, ApJ, 659, 1153

Wise, M. W., de Vries, M. N., Nulsen, P. E. J., et al. 2018, MNRAS

Zhuravleva, I., Churazov, E., Arévalo, P., et al. 2015, MNRAS, 450, 4184 